\section{P612 THE USE OF PHYSICAL RESTRAINTS ON CHILDREN: A RE-EVALUATION AFTER A DECADE}

Figen Işık Esenay ${ }^{*}$, Aslı Tufan Sezer, Ayten Demir. Ankara University, Ankara, Turkey

\subsection{6/archdischild-2019-epa.944}

Backround Physical restraint (PR), although controversial, is perceived as necessary for safe medical procedures. But also, this practice has important physical, psychological, social, ethical, legal and human rights consequences. In Turkey, pediatric nurses have been shown to use the restriction frequently.

Aim This study aimed to re-evaluate Turkish pediatric nurses' views and attitudes towards physical constraints after a decade.

Method This descriptive and cross-sectional study was carried out in two major hospitals in a capital city of Turkey between June-December 2018. The sample of the study consisted of pediatric units of 2 hospitals where the previous study was performed. All pediatric nurses were included in the study. A questionnaire based on to the previous study was used (Demir, 2007). The questionnairre consisted of two sections: pediatric nurses demographics and ideas and attitudes towards PR, the consent obtained, PR types used and complications.

Findings According to the study, nurses applied physical restraint to an average of 3.21 patients over the past week. This rate is lower than the previous study and this difference is statistically significant $(\mathrm{p}<0.05)$. The most commonly used PR methods in 2007 and 2018 are wrist (96.7\%; 44.9\%), ankle $(81 \% ; 28.8 \%)$, chest restraint by sheet $(14 \% ; 7,6 \%)$, respectively. There has also been a significant decrease in the rate of PR-related complications in parallel to the decrease in PR application rates and increased awareness of patient safety $(2,3 \%$ and $23,2 \%$, respectively). In the previous study, none of the nurses had received written or verbal consent from children or parents, while in the current study, $19.7 \%$ of nurses reported that they received consent from the child or their parents. In 2018, education and clinics are still associated with higher PR rates. It was determined that there is still no PR procedure in most of the pediatric clinics $(100 \%$ and $50,7 \%$ in 2007 and 2018, respectively). 83,3\% of nurses reported that nurse shortages were the main reason for increased PR usage.

Conclusion Although there is a decrease in the rate of usage of physical restraints by Turkish pediatric nurses, important issues such as obtaining informed consent, informing and establishing protocols related to PR are still not at the desired level. Actions are needed to reduce the use and complications of physical restrictions including the attention of nurse staffing and education on the use of restrictions.

\section{P614 ECONOMIC EVALUATIONS OF BLOOD PRESSURE MONITORING TECHNIQUES- A REVIEW OF METHODOLOGICAL ISSUES}

1,2Irum Awan*, ${ }^{3}$ Niklas Zethraeus. ' Karolinska Institutet, Stockholm, Sweden; ${ }^{2}$ Mercy University Hospital, Cork, Ireland; ${ }^{3}$ Department of LIME, Karolinska Institutet, Stockholm, Sweden

\subsection{6/archdischild-2019-epa.945}

Introduction The continued advancement in blood pressure monitoring techniques for hypertension raises the economic concerns. This signifies the value and importance of high quality economic evaluations as a guide for an efficient allocation of scarce resources and making reimbursement decisions.

Aim To assess different methods for the economic evaluation of blood pressure monitoring techniques in hypertension with their strengths and limitations and to suggest potential ways to improve the quality of economic evaluation in this field.

Methods A critical literature review of methods used in economic evaluation in blood pressure monitoring techniques was done. A literature search was conducted in selected databases to identify the full economic evaluations, cost-analysis and cost-consequence analyses, earliest from 1990s to date. The quality assessment of the methods used was done based on the standard Drummond's guidelines and Philip's checklists.

Results Eighteen unique peer reviewed economic evaluations were identified in blood pressure monitoring techniques. The most frequent type of economic evaluation was cost-effectiveness analysis from healthcare perspective and Markov cohort modelling. The quality of the methods used needs improvement in different areas, mainly the study design, valuation of cost and outcome and uncertainty analysis. Overall, results of economic evaluations are to be applied with caution to other settings.

Conclusions There is a constant need to improve the magnitude and quality of economic evaluations in this health technology, especially for children, that should follow standard quality criteria. More and more economic evaluations need to focus on subgroups with increased risk for target organ damage to heart, kidney and brain, subsequent to hypertension. The care providers and policy makers need to give due consideration to the quality of these health economic evaluations in making evidence-based decisions for patients and society as a whole.

\section{P615 PARENTS' OPINION ON VACCINATION}

Bella Matsukatova, Zarnigar Gumbatova, Alfia Aminova*, Tatyana Eryushova, Galina Borodina, Anna Procenko, Anastasia Platonova. Federal State Autonomous Educational Institution of Higher Education I.M. Sechenov First Moscow State Medical University of the Ministry of Health of the Russian Federation (Sechenov University), Moscow, Russian Federation, Moscow, Russian Federation

\subsection{6/archdischild-2019-epa.946}

Background The objective was to assess modern parents' attitude and the degree of their awareness of vaccination issues using a specially designed questionnaire. The vaccination issues are receiving close attention as scientists so medical practitioners. To study of the reasons for the rejection of vaccination has become particularly relevant in recent decades due to the anti-vaccination movement and outbreak in some countries.

Materials and methods The anonymous survey of 360 parents aged from 19 to 40 years old who were taking care of their children undergoing inpatient treatment was carried out. The selection of respondents was carried out by a random, continuous method. The questionnaire contained 34 questions with suggested answers to questions regarding parents' awareness and their attitude towards vaccinations in general.

Results The survey findings showed that $78,3 \%$ children were partly or fully vaccinated, whereas $21,7 \%$ children were not vaccinated. A total of $79 \%$ of parents believe that 